\title{
Bases moleculares de la resistencia a meticilina en Staphylococcus aureus
}

\author{
Alejandro Aguayo-Reyes, Mario Quezada-Aguiluz, Sergio Mella, Gisela Riedel, Andrés Opazo-Capurro, \\ Helia Bello-Toledo, Mariana Domínguez y Gerardo González-Rocha
}

\section{Molecular basis of methicillin-resistance in Staphylococcus aureus}

Staphylococcus aureus isolates resistant to several antimicrobials have been gradually emerged since the beginning of the antibiotic era. Consequently, the first isolation of methicillin-resistant $S$. aureus occurred in 1960 , which was described a few years later in Chile. Currently, S. aureus resistant to antistaphylococcal penicillins is endemic in Chilean hospitals and worldwide, being responsible for a high burden of morbidity and mortality. This resistance is mediated by the expression of a new transpeptidase, named PBP2a or PBP2', which possesses lower affinity for the $\beta$-lactam antibiotics, allowing the synthesis of peptidoglycan even in presence of these antimicrobial agents. This new enzyme is encoded by the mecA gene, itself embedded in a chromosomal cassette displaying a genomic island structure, of which there are several types and subtypes. Methicillin resistance is mainly regulated by an induction mechanism activated in the presence of $\beta$-lactams, through a membrane receptor and a repressor of the gene expression. Although mec-independent methicillin resistance mechanisms have been described, they are clearly infrequent.

Key words: Staphylococcus aureus, methicillin-resistance, methicillin-resistant Staphylococcus aureus (MRSA), antibiotic resistance.

Palabras clave: Staphylococcus aureus, resistencia a meticilina, Staphylococcus aureus resistente a meticilina (SARM), resistencia a antimicrobianos.

\section{Introducción}

$\mathrm{H}$ istóricamente, Staphylococcus aureus ha tenido un rol protagónico en una amplia variedad de cuadros clínicos, tales como infecciones de piel y tejidos blandos, neumonías, endocarditis, infecciones del torrente sanguíneo y osteomielitis, entre otros ${ }^{1}$. Tras la masificación del uso de penicilina se fueron seleccionando progresivamente cepas resistentes a antimicrobianos con actividad antiestafilocóccica ${ }^{2}$, lo que ha complejizado el tratamiento de las infecciones causadas por este patógeno.

En 1960 en Guildford (Inglaterra) se describió el primer aislado de $S$. aureus resistente a meticilina ${ }^{3}$ (SARM), que correspondía a la primera penicilina resistente a penicilinasa que, por lo demás, había sido recientemente introducida al arsenal terapéutico. Esto marcó el inicio de la descripción de este patógeno a nivel global ${ }^{4}$, siendo responsable, gradualmente de una gran carga de morbimortalidad en todo el mundo. En Chile, Ledermann $(1970)^{5}$ objetivó la progresiva disminución de la susceptibilidad a meticilina de $S$. aureus en la década de 1960, además de identificar una cepa aislada en 1967 con concentración inhibitoria mínima (CIM) de meticilina mayor a 6,25 $\mu \mathrm{g}$ / $\mathrm{mL}$ y catalogada como resistente. Sin embargo, no es hasta la década de 1980 en que realmente SARM comenzó a cobrar mayor relevancia en nuestro país ${ }^{6}$.
Si bien en Chile se han descrito otras cepas estafilocóccicas relevantes desde un punto de vista epidemiológico, tal como fue el primer aislado de $S$. aureus heteroresistente a vancomicina en $2015^{7}$, es SARM el verdadero protagonista de nuestra práctica clínica habitual. Por otro lado, las cefalosporinas de $5^{\mathrm{a}}$ generación han surgido como una nueva alternativa terapéutica aprobada por las agencias reguladoras correspondientes, contra algunas infecciones por SARM, y conocer los mecanismos moleculares que determinan la resistencia a meticilina ayuda a comprender mejor el funcionamiento de esta clase de antimicrobianos, que esperamos, estén pronto a disposición de los médicos clínicos.

En este artículo de revisión se abordarán los aspectos históricos más importantes de $S$. aureus en la era antimicrobiana, los mecanismos moleculares más relevantes que determinan la resistencia a meticilina, las distintas estructuras genéticas en las que puede residir el gen responsable de ésta y, finalmente, su mecanismo de regulación.

\section{Staphylococcus aureus en la era antimicrobiana}

Desde la introducción de la penicilina en 1928, y principalmente tras la masificación de su uso en la década
Universidad de Concepción, Concepción, Chile.

Facultad de Ciencias Biológicas, Departamento de Microbiología, Laboratorio de Investigación en Agentes Antibacterianos (AA-R, MQ-A, AO-C, HB-T, MD, GG-R) Facultad de Medicina, Departamento de Medicina Interna (AA-R, SM, GR) y Unidad de Infectología, Hospital Regiona "Dr. Guillermo Grant Benavente", Concepción, Chile (SM, GR)

Sergio Mella M. ha recibido honorarios como conferencista de Laboratorios Pfizer y MSD. Los otros autores no tienen conflictos de interés

Parte del trabajo fue financiado por el Proyecto Postdoctorado FONDECYT No 3150286.

Recibido: 24 de julio de 2017 Aceptado: 18 de diciembre de 2017

Correspondencia a: Gerardo González-Rocha ggonzal@udec.cl 
de 1940 , se fueron seleccionando gradualmente cepas resistentes a los distintos antibacterianos con actividad antiestafilocóccica². En 1944 ya se describía resistencia a penicilina, mediada por un mecanismo enzimático, específicamente una $\beta$-lactamasa con actividad de penicilinasa codificada en el gen $b l a_{z}$ y ubicado en un plásmido, capaz de hidrolizar el anillo $\beta$-lactámico y con esto inactivar el antimicrobiano ${ }^{8}$. En un comienzo, la resistencia a penicilina estuvo limitada a los hospitales, pero en la década de 1950 ya había evidencia de diseminación global ${ }^{2}$.

En este contexto, en 1959 surge la primera penicilina sintética resistente a la penicilinasa producida por $S$. aureus, meticilina. Esta molécula se convirtió en la precursora del conocido grupo de las penicilinas antiestafilocóccicas, cuyas modificaciones químicas permitieron resistir la acción de esta $\beta$-lactamasa. Específicamente, meticilina posee un mayor tamaño molecular que penicilina, a expensas del grupo dimetoxibenzoil añadido al ácido 6-aminopenicilánico (como radical R1), lo que confiere resistencia a la acción hidrolítica de la penicilinasa por impedimento estérico ${ }^{9}$. La penicilina antiestafilocóccica más usada en Chile es cloxacilina, que si bien químicamente es una isoxazolil-penicilina, es comparable a meticilina al ser una molécula químicamente más voluminosa a expensas, en este caso, del radical isoxazolil ${ }^{10}$ (Figura 1).

Transcurrido apenas un año desde la introducción de meticilina al uso clínico, se describió en Inglaterra, en 1960, el primer $S$. aureus resistente a dicho antimicrobiano, mediado por un mecanismo independiente de la hidrólisis enzimática y que confería resistencia a todos los $\beta$-lactámicos ${ }^{3}$. Sólo con el paso de los años se pudo dilucidar el gen responsable y la estructura genética que lo portaba. Esta nueva ventaja evolutiva permitió a SARM diseminarse por todo el mundo ${ }^{4}$, en un principio eminentemente a nivel hospitalario, en pacientes que compartían algunos factores de riesgo, tales como estadía en unidades de cuidados intensivos, haber sido sometidos a procedimientos invasores, ser pacientes en diálisis o portadores de dispositivos médicos ${ }^{11}$.

No obstante, en la década de 1990 comienza la descripción de casos genuinos de infecciones por SARM, pero de adquisición comunitaria y sin los factores de riesgo anteriormente descritos. La alarma se dio en Australia occidental, específicamente en población aborigen que no tenía contacto estrecho con centros médicos y que vivía en zonas relativamente aisladas ${ }^{12}$. Posteriormente, el CDC (Centers for Disease Control and Prevention) de Atlanta, Estados Unidos de América (E.U.A.), comunicó la muerte de cuatro niños sanos a causa de sepsis o neumonía necrosante por SARM adquiridos en la comunidad, ocurridas en el período 1997-99 ${ }^{13}$. Esto marcó el inicio de la diseminación de este patógeno comunitario a nivel global ${ }^{14}$. De hecho, en 2005 se publicó el primer caso en Sudamérica ${ }^{15}$ y en 2008 en Chile ${ }^{16}$. Es necesario aclarar en este punto que, si bien la resistencia a meticilina se ha informado en infecciones comunitarias en nuestro país, estos casos representan una minoría en comparación a los causados por cepas susceptibles a penicilinas antiestafilocóccicas (habitualmente cloxacilina como ya se mencionó), por lo que el problema de la resistencia en Chile se concentra de forma prevalente a nivel hospitalario.

Sin lugar a duda, $S$. aureus es la bacteria patógena grampositiva más relevante al que los médicos clínicos se enfrentan en la práctica infectológica habitual. Es así como el Ministerio de Salud, en su último informe de vigilancia de infecciones asociadas a la atención en salud (IAAS), cataloga a la etiología estafilocóccica como la más frecuente en una diversidad de entidades, tales como infección de sitio quirúrgico (de distintas localizaciones anatómicas), infección del torrente sanguíneo y neumonía asociada a ventilación mecánica, entre otros ${ }^{17}$. Por otro lado, el Grupo Colaborativo de Resistencia Bacteriana de la Sociedad Chilena de Infectología, en su último informe de susceptibilidad antimicrobiana de nuestro país, informa susceptibilidad a cloxacilina en apenas $51 \%$
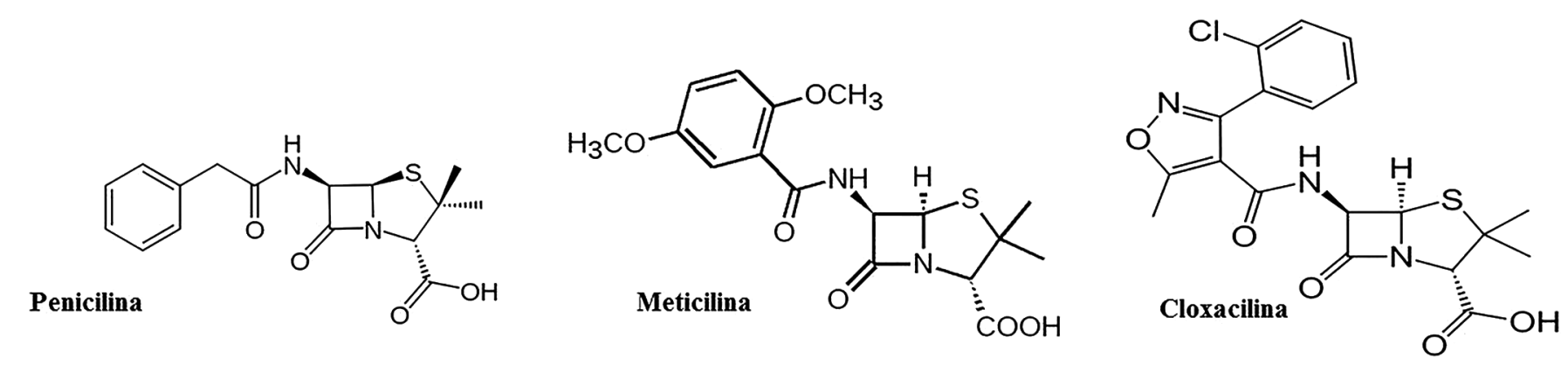

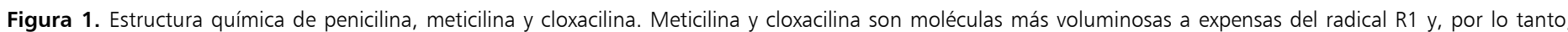
resistentes a la penicilinasa producida por Staphylococcus aureus. 

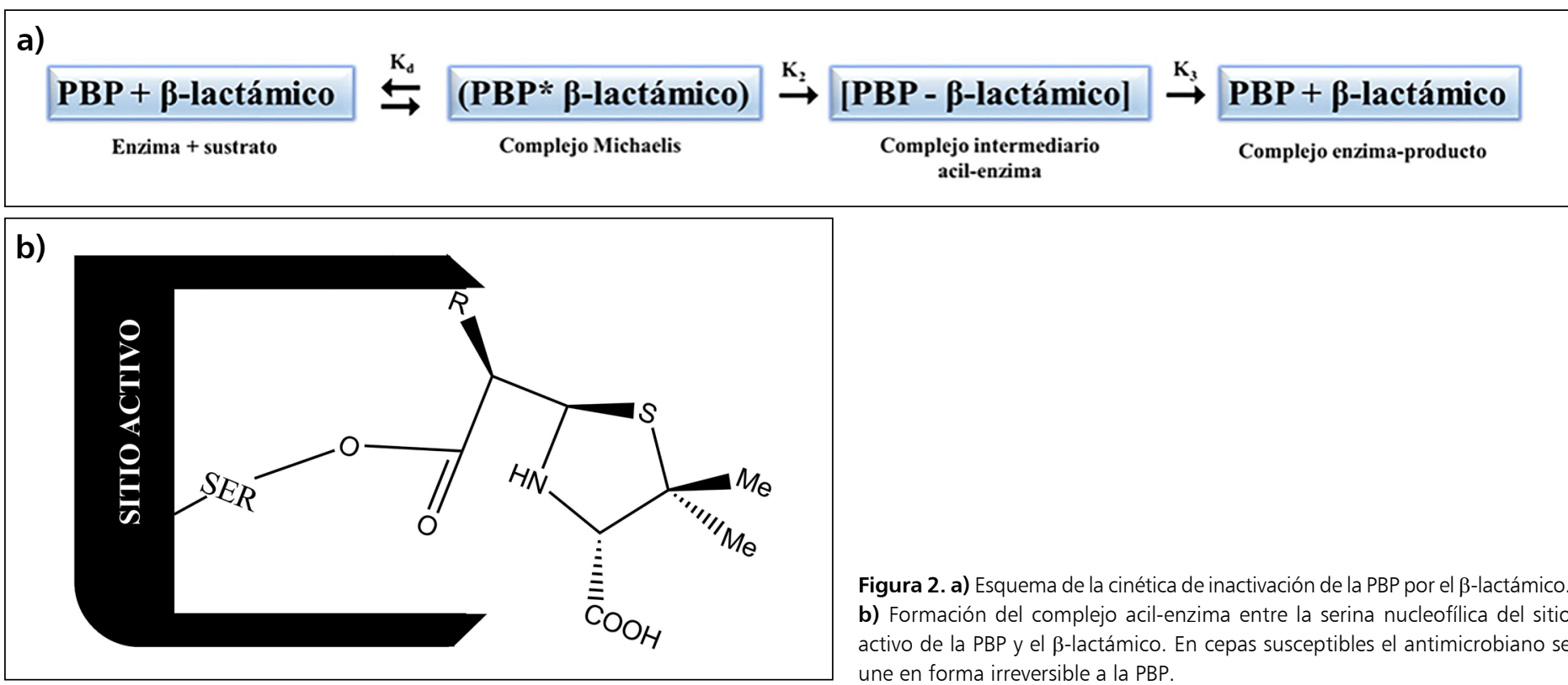

Figura 2. a) Esquema de la cinética de inactivación de la PBP por el $\beta$-lactámico. b) Formación del complejo acil-enzima entre la serina nucleofílica del sitio activo de la PBP y el $\beta$-lactámico. En cepas susceptibles el antimicrobiano se une en forma irreversible a la PBP.

de los aislados en población adulta ${ }^{18}$, lo que convierte a SARM en un microorganismo muy frecuente en los hospitales de Chile.

\section{Proteínas fijadoras de penicilina en $S$. aureus resistente a meticilina}

Es necesario recordar que la proteína blanco de acción de los $\beta$-lactámicos se conoce universalmente como PBP (del inglés penicillin-binding protein). Las PBPs corresponden a enzimas, principalmente con función transpeptidasa, que participan en el entrecruzamiento de las hebras de peptidoglicano (PG), específicamente entre las cadenas laterales de naturaleza aminoacídica que están unidas al ácido $\mathrm{N}$-acetilmurámico ${ }^{19}$. Con este entrecruzamiento de hebras de PG la bacteria logra resistencia frente a la lisis osmótica. Es precisamente esta etapa de síntesis la que inhibe el $\beta$-lactámico (la etapa extracelular), aprovechándose de su homología estructural con el substrato natural de las PBPs, el dipéptido terminal D-alanina-D-alanina ${ }^{20}$. En otras palabras, la PBP (transpeptidasa) forma un complejo acil-enzima con el $\beta$-lactámico y no con el dipéptido (su substrato natural), intermediario que, por lo demás, es sumamente estable, lo que determina una inhibición irreversible de la PBP (Figura 2a). La estabilidad del complejo acil-enzima entre la PBP y el antimicrobiano viene determinado molecularmente, ya que el anillo $\beta$-lactámico ocupa el sitio activo de la enzima e impide que se acomode en éste una molécula aceptora del grupo acilo o una potencial molécula de agua que permita la hidrólisis del complejo ${ }^{21}$ (Figura 2b).
Todas las cepas de $S$. aureus poseen cuatro tipos de PBPs: PBP1, PBP2, PBP3 y PBP4. Las primeras tres tienen función transpeptidasa y corresponden a PBPs de alto peso molecular, siendo PBP2 la única bifuncional, es decir, además posee función transglicosilasa. PBP4 es la única de bajo peso molecular y participa en procesos de división, remodelado y reciclado de PG. Ninguna de estas PBPs nativas tiene regulación alostérica ${ }^{22}$.

El mecanismo más importante y frecuente que determina la resistencia a meticilina es la adquisición de una nueva PBP, denominada PBP2a o PBP2'23, de la cual queremos resaltar dos propiedades distintivas. La primera es la baja afinidad que tiene esta nueva transpeptidasa por el antimicrobiano $\beta$-lactámico, lo que está determinado por la configuración de su sitio activo que es menos accesible al antimicrobiano al tener la hendidura más estrecha $^{24}$ (Figura 3). Lo anterior determina que con las concentraciones que alcanza el $\beta$-lactámico in vivo no se forma el intermediario acil-enzima entre el antimicrobiano y la PBP2a, por lo que la función de transpeptidación de la enzima se mantiene, permitiendo el entrecruzamiento de hebras de $\mathrm{PG}^{21}$.

La segunda característica distintiva de la PBP2a es su sitio de regulación alostérico, al que podría unirse $\mathrm{PG}$ en formación, lo que favorecería la apertura del sitio activo para dejar mejor posicionado el residuo de serina nucleofílico que participa en la formación del enlace peptídico. Lo anterior tiene bastante lógica, ya que el sitio activo de la PBP sería más accesible sólo en presencia de PG naciente que debe ser transpeptidado ${ }^{22}$.

En suma, si una cepa de SARM es expuesta a meticilina o cloxacilina, el antimicrobiano es capaz de inhibir in vivo 


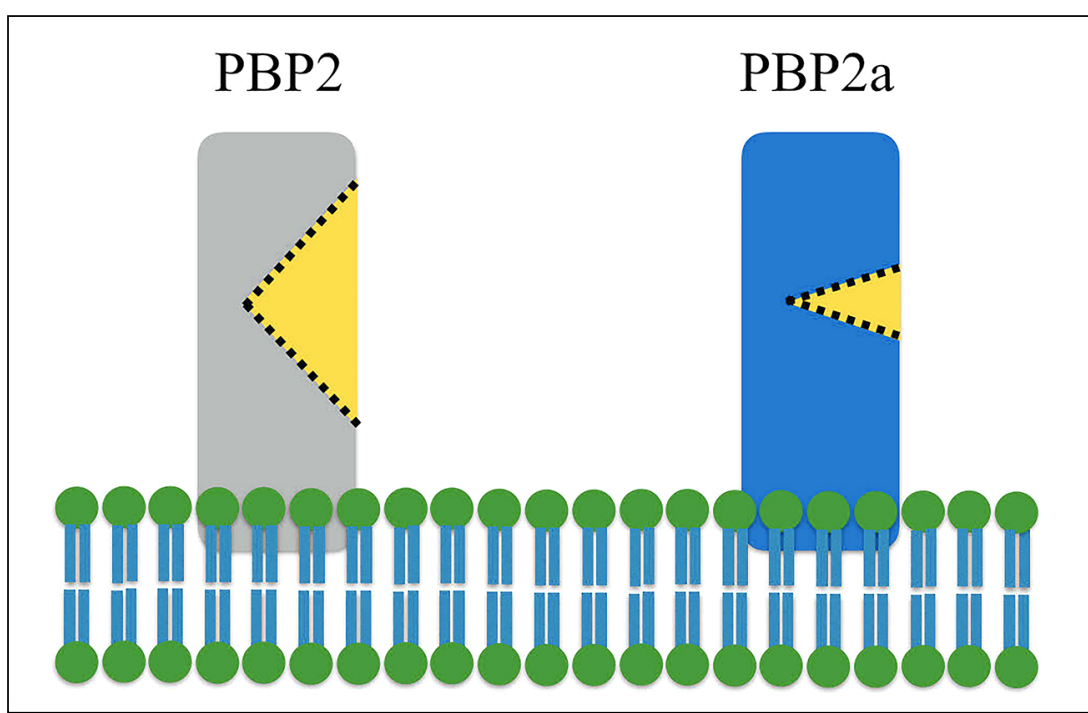

Figura 3. Esquema comparativo de los dominios transpeptidasa de PBP2 y PBP2a. Destaca la configu ración más estrecha del sitio activo de la PBP2a, responsable de la menor afinidad por el $\beta$-lactámico.

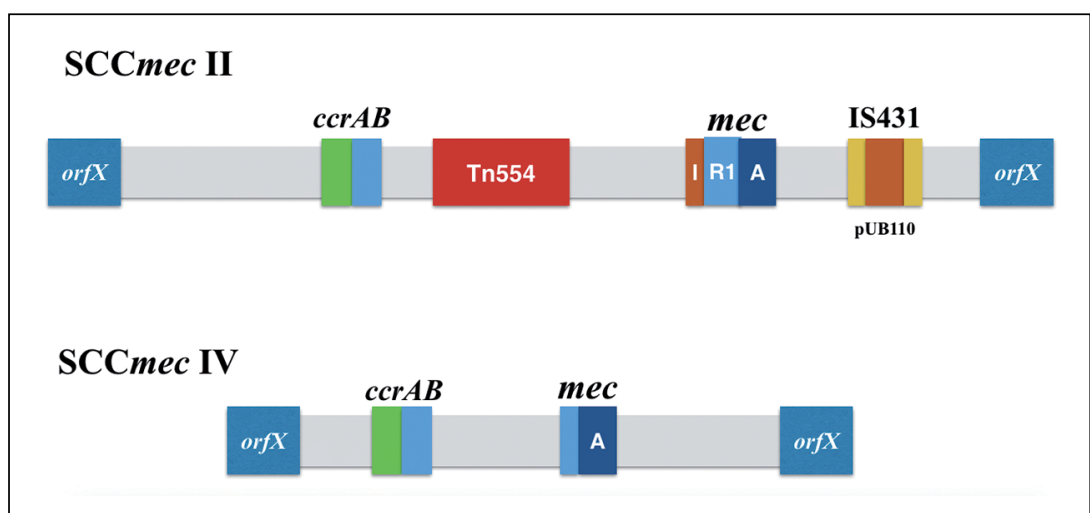

Figura 4. Estructura general del cassette cromosomal estafilocóccico SCCmec II y IV. Adaptado de Nat Rev Microbiol 2009; 7 (9): 629-41.

las PBPs nativas de la bacteria (PBP 1-4), pero no logra inhibir a la PBP2a, manteniendo el entrecruzamiento del PG en presencia de cualquier $\beta$-lactámico, exceptuando por supuesto, las cefalosporinas de $5^{\text {a }}$ generación ${ }^{25}$.

\section{La PBP2a se encuentra codificada en el gen mecA}

Staphylococcus aureus resistente a meticilina posee el gen $m e c A$, que codifica la PBP2a, responsable de la resistencia a meticilina. A su vez, este gen se encuentra inserto en una isla genómica, denominada $\mathrm{SCCmec}$, del inglés Staphylococcal Cassette Chromosome mec, un elemento genético móvil ${ }^{26}$. Se ha propuesto que este gen podría haber residido inicialmente en especies de Staphylococcus coagulasa negativa, como $S$. sciuri o $S$. fleuretti (ambos relacionados con animales) y desde éstos haberse transferido a $S$. aureus $^{27}$.
Existen varios tipos y subtipos de SCCmec, pero todos comparten algunas características ${ }^{28}$ que se esquematizan en la Figura 4. Estos cassettes se integran en el cromosoma junto al gen $\operatorname{orf} X$, que codifica para una metiltransferasa ribosomal, y todos poseen un complejo de genes mec, compuesto por mecA (que codifica la PBP2a) y los genes mecI y mecR cuyos productos regulan la expresión de la resistencia a meticilina. Adicionalmente, los SCCmec poseen un complejo de genes $c c r$, compuesto por uno o dos genes que codifican recombinasas sitio específicas responsables de la movilidad del cassette cromosomal. Otra región importante son las denominadas regiones $\mathrm{J}$ (del inglés joining regions) que corresponden a componentes no esenciales del cassette y que pueden contener genes de resistencia para otras familias de antimicrobianos (macrólidos, lincosamidas y aminoglucósidos-aminociclitoles) y para metales pesados. Finalmente, forman parte de esta estructura genética las secuencias repetidas en los extremos, que son reconocidas por las recombinasas para la inserción/ escisión del elemento genético móvil.

Las distintas estructuras genéticas del complejo de genes mec y del complejo de genes $c c r$ determinan el tipo de SCCmec y, por otro lado, las diferencias en las regiones $\mathrm{J}$ determinan los subtipos dentro de un mismo cassette cromosomal ${ }^{29}$. Tal como se muestra en la Tabla 1 , SCCmec I y IV poseen el mismo complejo de genes mec clase B (IS1272- $\Delta m e c R 1-m e c A$-IS431), pero diferente complejo de genes $c c r$, siendo tipo 1 para SCCmec I y tipo 2 para el SCCmec IV, lo que se basa en la distinta identidad de las secuencias nucleotídicas de los genes que codifican las recombinasas. A la fecha se han descrito 11 tipos de cassettes cromosomales estafilocóccicos ${ }^{28}$, los que quedan resumidos en la Tabla 1.

De acuerdo a la epidemiología molecular de SARM, se ha podido determinar que en distintas localizaciones geográficas existe predominancia de distintos $\mathrm{SCC}_{\mathrm{Cec}}{ }^{28}$. Es así como el primer SARM descrito en Inglaterra (clon arcaico) poseía el SCCmec I, pero fue desplazado a partir de la década de 1980 por un clon de SARM que poseía el cassette cromosomal tipo $\mathrm{II}^{30}$. Por su parte, en E.U.A. predomina el cassette II y IV ${ }^{2}$. En Chile, un estudio realizado en Valdivia muestra un claro predominio del SCCmec I a nivel hospitalario ${ }^{31}$, lo que es compatible con resultados obtenidos en nuestro laboratorio ${ }^{32}$, aunque también identifica cepas portadoras del SCCmec IV. El clon chileno/cordobés ha demostrado gran estabilidad en nuestro país, y es por amplio margen el predominante en los aislados clínicos de pacientes hospitalizados en nuestro territorio. Característicamente posee el SCCmec I, pertenece al ST5 y es susceptible a glucopéptidos, oxazolidinonas, tetraciclinas, rifampicina y cotrimoxazol. Es más, su éxito biológico le había permitido diseminarse por gran parte de Sudamérica; no obstante, el surgimiento de 
nuevos clones biológicamente más exitosos ha favorecido la predominancia de nuevos linajes en otros países latinoamericanos, tales como el USA 300-LV en Colombia, Ecuador y Venezuela y el ST30-SCCmec IVc, además del ST5-SCCmec IVa en Argentina ${ }^{33}$.

El programa de vigilancia de SARM con fenotipo comunitario del Instituto de Salud Pública, si bien incluye la detección de los genes mecA, pvl (toxina de Panton-Valentine) y su tipificación por metodologías de macrorestricción, Spa typing y MLST, no incluye la identificación del tipo de cassette cromosomal (http:// www.ispch.cl/content/19540).

Es interesante analizar las diferencias en las estructuras genéticas de un cassette cromosomal estafilocóccico clásicamente hospitalario versus uno comunitario. El primero cuenta con determinantes adicionales de resistencia a otras familias de antibacterianos, configurando un clásico fenotipo de multi-resistencia. Es así como el SCCmec II es un cassette más grande que posee el transposón Tn554 en la región J2, el que es responsable de la resistencia a macrólidos, lincosamidas y estreptograminas del grupo $\mathrm{B}$, y además, contiene el plásmido pUB110 integrado en la región J3, que determina resistencia a tobramicina ${ }^{2}$. Por otro lado, SCCmec IV es una estructura más simple y que sólo porta el gen de resistencia $m e c A$, por lo que habitualmente las cepas que tienen este cassette sólo son resistentes a $\beta$-lactámicos ${ }^{34}$. Es importante mencionar, que la separación entre SARM hospitalarios y comunitarios está poco a poco perdiendo validez, al menos en países con alta prevalencia de ambos patógenos. Lo anterior se debe a la creciente descripción de cepas de SARM clásicamente comunitarias en los hospitales, desplazando a otros

\begin{tabular}{|c|c|c|c|c|}
\hline SCCmec & $\begin{array}{l}\text { Complejo de } \\
\text { genes mec }\end{array}$ & $\begin{array}{l}\text { Estructura del complejo } \\
\text { de genes mec }\end{array}$ & $\begin{array}{l}\text { Complejo de } \\
\text { genes ccr }\end{array}$ & Genes ccr \\
\hline I & Clase B & 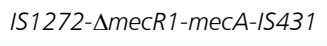 & Tipo 1 & cCrA1, $\mathrm{CCrB1}$ \\
\hline II & Clase A & mecl-mecR1-mecA-IS431 & Tipo 2 & cCrA2, $\mathrm{ccrB2}$ \\
\hline III & Clase A & mecl-mecR1-mecA-IS431 & Tipo 3 & cCrA3, ccrB3 \\
\hline IV & Clase B & 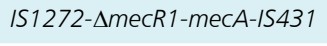 & Tipo 2 & cCrA2, $\mathrm{ccrB2}$ \\
\hline$\vee$ & Clase C2 & IS431-mecA- $\Delta$ mecR1-IS431 & Tipo 5 & $\operatorname{ccrCl}$ \\
\hline $\mathrm{VI}$ & Clase B & 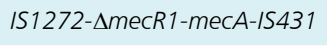 & Tipo 4 & сCrA4, сCrB4 \\
\hline VII & Clase C1 & IS431-mecA- $\Delta$ mecR1-IS431 & Tipo 5 & $\operatorname{ccrCl}$ \\
\hline VIII & Clase A & mecl-mecR1-mecA-IS431 & Tipo 4 & cCrA4, сcrB4 \\
\hline IX & Clase C2 & IS431-mecA- $\Delta$ mecR1-IS431 & Tipo 1 & cCrA1, $\mathrm{cCr} B 1$ \\
\hline$x$ & Clase C1 & IS431-mecA- $\Delta$ mecR1-IS431 & Tipo 7 & c crA1, c crBb \\
\hline$X I$ & Clase E & blaz-mecc-mecR1-mecl & Tipo 8 & $\triangle C r A 1, \quad c c r B 3$ \\
\hline
\end{tabular}

Adaptado de Liu et al. 2016. Microb Pathog 2016;101:56-67.

clones nosocomiales menos exitosos ${ }^{35}$. No obstante, en países con baja prevalencia de SARM de la comunidad la distinción aún podría ser útil. Cabe destacar, que la determinación del SCCmec como único blanco para la detección de resistencia a meticilina no se recomienda, ya que se han descrito elementos que no portan el gen $m e c A$, pero contiene otros genes que codifican factores de virulencia o resistencia a otros antimicrobianos y metales pesados ${ }^{36}$.

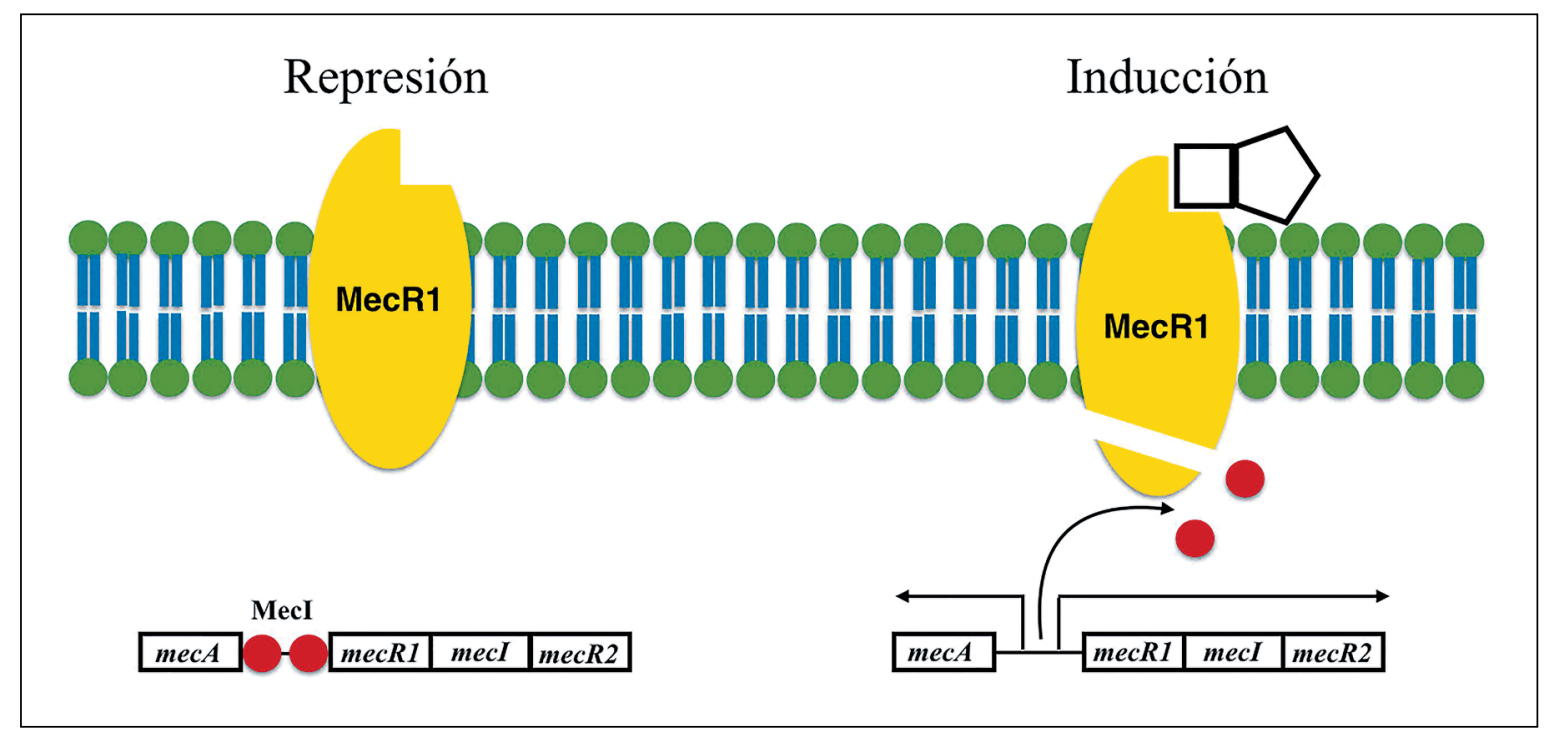

Rev Chilena Infectol 2018; 35 (1): 7-14
Figura 5. Regulación de la resistencia a meticilina. En ausencia del antimicrobiano, Mecl actúa como represor del complejo de genes mec tras unirse en forma de homodímero a la región operadora. En presencia del $\beta$-lactámico, éste se une al dominio PBP de MecR1 el que se activa y gatilla la ruptura de Mecl, liberando la represión y permitiendo de esta forma la expresión de los genes del complejo mec. 


\section{Regulación de la resistencia a meticilina}

Esta regulación es homóloga a la descrita para la penicilinasa estafilocóccica y se basa eminentemente en un receptor de membrana (sensor del $\beta$-lactámico) y un represor de la expresión del gen ${ }^{37}$. Cuando nos referimos previamente al complejo de genes $m e c$, aparte de $m e c A$, nombramos a sus genes reguladores: $m e c R$ y $m e c I$, y en este ítem ambos cobran relevancia. Tanto mecA como sus genes reguladores se encuentran adyacentes, pero se transcriben en forma divergente ${ }^{21}$.

En ausencia del $\beta$-lactámico, MecI actúa como homodímero y reconoce una secuencia palindrómica de 30 $\mathrm{pb}$ ( $15 \mathrm{pb}$ en díada simétrica), abarcando las secuencias promotoras - 10 de $m e c A$ y -35 de $m e c R 1$, lo que interfiere con la unión de la ARN polimerasa y, por lo tanto, con la expresión de la resistencia a meticilina ${ }^{21}$.

En presencia del $\beta$-lactámico, éste se une al dominio PBP de la proteína de membrana MecR1, lo que desencadena la activación autolítica de su dominio metaloproteinasa. Luego, no se sabe si en forma directa o indirecta, MecR1 rompe el homodímero MecI (el represor) entre sus residuos asparragina 101 y fenilalanina 102 , lo que desestabiliza la dimerización de esta proteína represora y libera la represión del complejo de genes $m e c$, favoreciendo por lo tanto la expresión de mecA y de la transpeptidasa PBP2a, determinante de la resistencia a meticilina ${ }^{38}$. La desrepresión de los genes regulatorios favorece también la síntesis de la proteína MecR2, con función antirrepresora, ya que es capaz de unirse a MecI e impedir que se una al promotor de mecA, además de favorecer su degradación por proteasas citoplasmáticas ${ }^{39}$. En la Figura 5 se resumen los aspectos más relevantes de la regulación de la resistencia a meticilina en $S$. aureus.

Por último, existen genes no codificados en el cassette cromosomal, es decir, que están localizados en otros lugares del cromosoma bacteriano, pero de igual forma influyen en la resistencia a meticilina. A estos genes se les llamó inicialmente fem (del inglés factors essential for methicillin resistance) y están asociados a la síntesis del $\mathrm{PG}^{40}$.

\section{Otros mecanismos de resistencia a meticilina}

En el año 2011 fue informado en la cepa de $S$. aureus LGA251 el último de los cassettes cromosomales descritos a la fecha ${ }^{41}$, SCCmec XI, el que tiene algunas particularidades dignas de resaltar. En primer lugar, el complejo de genes mec incluye el gen $b a_{z}$ (penicilinasa), configurando una nueva clase denominada E. Además, el gen $m e c A_{\mathrm{LGA}_{251}}$ tiene una homología de sólo $70 \%$ en la secuencia nucleotídica con el gen $m e c A$. Por esto la PBP que se sintetiza a partir de este gen, si bien es la responsable de la resistencia a meticilina, sólo tiene $63 \%$ de homología con la PBP2a. Posteriormente, el gen que codificaba para esta nueva transpeptidasa se renombró como $m e c C^{42}$. Las cepas portadoras de este determinante genético de resistencia se han asociado, principalmente, a la actividad veterinaria; no obstante, también ha sido descrito en cepas aisladas de seres humanos. En algunos países como Dinamarca, cepas de $S$. aureus portadoras de mec $C$ pueden ser responsables de hasta $2 \%$ de los casos de infecciones causadas por cepas resistentes a meticilina en humanos ${ }^{43}$. Es importante resaltar que la detección fenotípica y genotípica de la resistencia en $S$. aureus mediada por $m e c C$ tiene algunas particularidades. Los métodos automatizados, habitualmente usados en laboratorios clínicos tienen sensibilidades que oscilan entre $65-97 \%$, dependiendo del sistema utilizado. Por otro lado, el uso de discos de cefoxitina ha demostrado ser efectivo, simple y económico si lo comparamos con ensayos que utilizan oxacilina, al cual se le han atribuido un gran porcentaje de falsos negativos, invalidando la utilización de este último antimicrobiano como método único. Desde un punto de vista genotípico, la baja homología nucleotídica entre mecA y mecC obliga a usar partidores específicos para este último al utilizar reacción de polimerasa en cadena. Asimismo, los ensayos antigénicos específicos para detectar PBP2a no detectan la transpeptidasa codificada por $\mathrm{mecC}^{44}$. En suma, debemos estar atentos a los métodos utilizados en nuestros centros, para así evitar la posibilidad de obtener falsos negativos en la desafiante tarea de detectar resistencia a meticilina mediada por $m e c C$.

Existen, además, cepas de $S$. aureus denominadas BORSA (del inglés Borderline oxacillin-resistant $S$. aureus), cuyo mecanismo de resistencia se asocia a un aumento en la actividad $\beta$-lactamasa, llegando a CIM frente a oxacilina cercanas al punto de corte, es decir, presentan un bajo nivel de resistencia ${ }^{45}$.

Otra variedad de $S$. aureus que expresa bajo nivel de resistencia a meticilina, a través de un mecanismo independiente de $m e c$, son las cepas MOD-SA (del inglés modified $\mathrm{PBP}$ ), denominadas así por tener modificaciones en sus PBPs nativas, aparentemente por acumulación de mutaciones en los dominios transpeptidasa ${ }^{46}$.

Si bien los aislados que presentan estos últimos mecanismos pueden ser buenos ejemplos de la diversidad genética bacteriana que condiciona diferentes mecanismos de resistencia a un mismo antimicrobiano, es necesario recalcar que representan una minoría de los aislados en comparación a la amplia prevalencia, principalmente en medicina humana, de la resistencia a meticilina mediada por el gen $m e c A$.

\section{Conclusión}

La resistencia bacteriana a antimicrobianos es un fenómeno natural y la inmensa plasticidad genética de 
estos microorganismos es difícil de dimensionar en su totalidad. Con la masificación del uso de los antimicrobianos no hacemos más que seleccionar cepas resistentes, las que consecuentemente se convierten en la población predominante. Esto es exactamente lo que ha pasado con $S$. aureus, primero con penicilina y luego con meticilina. El mecanismo de resistencia de este patógeno a las penicilinas antiestafilocóccicas, tal como ocurre habitualmente en las bacterias grampositivas, tiene que ver con el sitio blanco de acción, en este caso a través de la síntesis de una nueva PBP que tiene baja afinidad por los $\beta$-lactámicos. El gen que codifica esta nueva transpeptidasa y sus genes reguladores se encuentran insertos dentro de una estructura versátil y móvil denominada cassette cromosomal estafilocóccico, del que existen varios tipos de acuerdo a su estructura genética. El predominio de SCCmec implica fenotipos de resistencia característicos, como el presente en SARM comunitarios donde el cassette sólo codifica resistencia a meticilina y carece de otros determinantes de resistencia. La epidemiología molecular sobre SARM en nuestro país es incipiente $\mathrm{y}$, en general, se limita a la evidencia del predominio del denominado clon chileno/ cordobés, cuya estructura y características están en creciente descripción junto con el aislamiento de variantes comunitarias resistentes a meticilina.

\section{Resumen}

Desde el inicio de la era antimicrobiana se han ido seleccionando gradualmente cepas de Staphylococcus aureus resistentes a antimicrobianos de amplio uso clínico. Es así como en 1960 se describen en Inglaterra las primeras cepas resistentes a meticilina, y algunos años después son informadas en hospitales de Chile. Actualmente, S. aureus resistente a penicilinas antiestafilocóccicas es endémico en los hospitales de nuestro país y del mundo, siendo responsable de una alta morbimortalidad. La resistencia es mediada habitualmente por la síntesis de una nueva transpeptidasa, denominada PBP2a o PBP2' que posee menos afinidad por el $\beta$-lactámico, y es la que mantiene la síntesis de peptidoglicano en presencia del antimicrobiano. Esta nueva enzima se encuentra codificada en el gen $m e c A$, a su vez inserto en un cassette cromosomal con estructura de isla genómica, de los cuales existen varios tipos y subtipos. La resistencia a meticilina se encuentra regulada, principalmente, por un mecanismo de inducción de la expresión del gen en presencia del $\beta$-lactámico, a través de un receptor de membrana y un represor de la expresión. Si bien se han descrito mecanismos generadores de resistencia a meticilina mec independientes, son categóricamente menos frecuentes.

\section{Referencias bibliográficas}

1.- Lowy F D. Staphylococcus aureus infections. N Engl J Med 1998; 339: 520-32.

2.- Chambers H F, Deleo F R. Waves of resistance: Staphylococcus aureus in the antibiotic era. Nat Rev Microbiol 2009; 7 (9): 629-41.

3.- Barber M. Methicillin-resistant staphylococci. J Clin Pathol 1961; 14: 385-93.

4.- Ayliffe G A. The progressive intercontinental spread of methicillin-resistant Staphylococcus aureus. Clin Infect Dis 1997; 24 Suppl 1: S749.

5.- Ledermann G W. Sensibilidad in vitro del $S$. aureus a meticilina y cloxacilina en los últimos ocho años (1961-1968). Rev Chil Pediatr 1970; 41: 213-5.

6.- Gil M. Staphylococcus aureus: Microbiología y aspectos moleculares de la resistencia a meticilina. Rev Chilena Infectol 2000; 17 (2): 145-52.

7.- Vega F, Alarcón P, Domínguez M, Bello H, Riedel G, Mella S, et al. Aislamiento de Staphylococcus aureus hetero-resistente a vancomicina en Hospital Clínico Regional de Concepción, Chile. Rev Chilena Infectol 2015; 32 (5): 588-90.

8.- Kirby W M. Extraction of a highly potent penicillin inactivator from penicillin resistant staphylococci. Science 1944; 99 (2579): 452-3.
9.- Mitscher L A, Lemke T L, Gentry E J. Chapter 38: Antibiotics and antimicrobial agents. Lemke T L, Williams D A, Roche V F and Zito SW's Foyes's Principles of Medicinal Chemistry, 6th ed. Philadelphia: Wolters Kluwer/Lippincott Williams \& Wilkins; 2008, p. 1028-82.

10.- Zemelman R, Norambuena R, Vergara L, Gacitua R. Los antibióticos $\beta$-lactámicos: agrupación según su estructura química y sus propiedades bacteriológicas. Rev Med Chile 1987; 115: 983-91.

11.- Deresinski S. Methicillin-resistant Staphylococcus aureus: an evolutionary, epidemiologic, and therapeutic odyssey. Clin Infect Dis 2005; 40 (4): 562-73.

12.- Udo E E, Pearman J W, Grubb W B. Genetic analysis of community isolates of methicillinresistant Staphylococcus aureus in Western Australia. J Hosp Infect 1993; 25 (2): 97-108.

13.- Centers for Disease Control and Prevention. Four pediatric deaths from community-acquired methicillin-resistant Staphylococcus aureusMinnesota and North Dakota, 1997-1999. JAMA 1999; 282 (12): 1123.

14.- DeLeo F R, Otto M, Kreiswirth B N, Chambers H F. Community-associated meticillin-resistant Staphylococcus aureus. Lancet 2010; 375 (9725): 1557-68

15.- Ribeiro A, Dias C, Cícera Silva-Carvalho M,
Berquó L, Ferreira F A, Neves R, et al. First report of infection with community-acquired methicillin-resistant Staphylococcus aureus in South America. J Clin Microbiol 2005; 43 (4): 1985-8.

16.- Noriega L M, González P, Hormazábal J C, Pinto C, Canals M, Munita J M, et al. Staphylococcus aureus comunitario resistente a cloxacilina: Comunicación de los primeros cinco casos descritos en Chile. Rev Med Chile 2008; 136 (7): 886-91.

17.- Informe de vigilancia de infecciones asociadas a la atención en salud 2014, http://web.minsal. cl/wp-content/uploads/2016/10/informeIAAS-2014.pdf (fecha acceso el 01 de julio de 2017).

18.- Cifuentes M, Silva F, García P, Bello H, Briceño I, Calvo M, et al. Susceptibilidad antimicrobiana en Chile 2012. Rev Chilena Infectol 2014; 31 (2): 123-30.

19.- Giesbrecht P, Kersten T, Maidhof H, Wecke J. Staphylococcal cell wall: morphogenesis and fatal variations in the presence of penicillin. Microbiol Mol Biol Rev 1998; 62 (4): 1371414.

20.- Tipper D J, Strominger J L. Mechanism of action of penicillins: a proposal based on their structural similarity to acyl-D-alanyl-D-alanine. Proc Natl Acad Sci U S A1965; 54 (4): 113341. 
21.- Peacock S J, Paterson G K. Mechanisms of methicillin resistance in Staphylococcus aureus. Annu Rev Biochem 2015; 84: 577-601.

22.- Acebrón I, Chang M, Mobashery S, Hermoso J A. The allosteric site for the nascent cell wall in penicillin-binding protein $2 \mathrm{a}$ :an Achilles' hell of methicillin-resistant Staphylococcus aureus. Curr Med Chem 2015; 22 (14): 1678-86.

23.- Chambers H F. Methicillin resistance in staphylococci: molecular and biochemical basis and clinical implications. Clin Microbiol Rev 1997; 10 (4): 781-91.

24.- Lim D, Strynadka N C. Structural basis for the beta lactam resistance of PBP2a from methicillin-resistant Staphylococcus aureus. Nat Struct Biol 2002; 9 (11): 870-6.

25.- Rodvold K A, Mcconeghy K W. Methicillinresistant Staphylococcus aureus therapy: Past, present, and future. Clin Infect Dis 2014; 58 (SUPPL. 1): 20-7.

26.- Aires De Sousa M, De Lencastre H. Bridges from hospitals to the laboratory: Genetic portraits of methicillin-resistant Staphylococcus aureus clones. FEMS Immunol Med Microbiol 2004; 40 (2): 101-11.

27.- Couto I, de Lencastre H, Severina E, Kloos W, Webster J A, Hubner R J, et al. Ubiquitous presence of a mecA homologue in natural isolates of Staphylococcus sciuri. Microb Drug Resist 1996; 2 (4): 377-91.

28.- Liu J, Chen D, Peters B M, Li L, Li B, Xu Z, et al. Staphylococcal chromosomal cassettes mec (SCCmec): A mobile genetic element in methicillin-resistant Staphylococcus aureus. Microb Pathog 2016; 101: 56-67.

29.- International Working Group on the Staphylococcal Cassettes Chromosome elements, http://www.sccmec.org (fecha de acceso el 01 de julio de 2017).

30.- Deurenberg R H, Vink C, Kalenic S, Friedrich A W, Bruggeman C A, Stobberingh E E. The molecular evolution of methicillin-resistant Staphylococcus aureus. Clin Microbiol Infect 2007; 13 (3): 222-35.
31.- Medina G, Egea A L, Otth C, Otth L, Fernández $\mathrm{H}$, Bocco J L, et al. Molecular epidemiology of hospital-onset methicillin-resistant Staphylococcus aureus infections in Southern Chile. Eur J Clin Microbiol Dis 2013; 32 (12): 1533-40.

32.- Vega F. Caracterización fenotípica y molecular de cepas de Staphylococcus aureus resistente a meticilina (SARM) aisladas en el Hospital "Dr. Guillermo Grant Benavente” de Concepción. Laboratorio de Investigación en Agentes Antibacterianos, Universidad de Concepción, Concepción, Chile, 2015. Tesis de Magíster en Ciencias mención Microbiología 71 pp.

33.- Arias C A, Reyes J, Carvajal L P, Rincón S, Díaz L, Panesso D, et al. A prospective cohort multicenter study of molecular epidemiology and phylogenomics of Staphylococcus aureus bacteremia in nine latin american countries. Antimicrob Agents Chemother 2017; 61 (10): 1-12.

34.- David M Z, Daum R S. Community-associated methicillin-resistant Staphylococcus aureus: Epidemiology and clinical consequences of an emerging epidemic. Clin Microbiol Rev 2010; 23 (3): 616-87.

35.- Kale P, Dhawan B. The changing face of community-acquired methicillin-resistant Staphylococcus aureus. Indian J Med Microbiol 2016; 34 (3): 275-85.

36.- International Working Group on the Classification of Staphylococcal Cassette Chromosome Elements (IWG-SCC). Classification of staphylococcal cassette chromosome mec (SCCmec): Guidelines for reporting novel $\mathrm{SCC} m e c$ elements. Antimicrob Agents Chemother 2009; 53 (12): 4961-7.

37.- Lowy F D. Antimicrobial resistance: the example of Staphylococcus aureus. J Clin Invest 2003; 111 (9): 1265-73.

38.- Arêde P, Oliveira D C. Proteolysis of mecA repressor is essential for expression of methicillin resistance by Staphylococcus aureus. Antimicrob Agents Chemother 2013; 57 (4): 2001-2.
39.- Arêde P, Milheiriço C, de Lencastre H, Oliveira D C, Coll M. The anti-repressor mecR2 promotes the proteolysis of the mecA repressor and enables optimal expression of $\beta$-lactam resistance in MRSA. PLoS Pathog 2012; 8 (7): e1002816.

40.- Berger-Bächi B, Rohrer S. Factors influencing methicillin resistance in staphylococci. Arch Microbiol 2002; 178 (3): 165-71.

41.- García-Álvarez L, Holden M T, Lindsay H, Webb C R, Brown D F, Curran M D, et al. Meticillin-resistant Staphylococcus aureus with a novel mecA homologue in human and bovine populations in the UK and Denmark: a descriptive study. Lancet Infect Dis 2011; 11 (8): 595-603.

42.- Ito T, Hiramatsu K, Tomasz A, de Lencastre $\mathrm{H}$, Perreten V, Holden M T, et al. Guidelines for reporting novel mecA gene homologues. Antimicrob Agents Chemother 2012; 56 (10): 4997-9.

43.- Petersen A, Stegger M, Heltberg O, Christensen J, Zeuthen A, Knudsen L K, et al. Epidemiology of methicillin-resistant Staphylococcus aureus carrying the novel mecC gene in Denmark corroborates a zoonotic reservoir with transmission to humans. Clin Microbiol Infect 2013; 19 (1): E16-22.

44.- Ford BA. mecC-MRSA: Hiding in plain sight J Clin Microbiol 2017;56(1). pii: e01549-17. doi: 10.1128/JCM.01549-17. Print 2018 Jan.

45.- McDougal L K, Thornsberry C. The role of beta-lactamase in staphylococcal resistance to penicillinase-resistant penicillins and cephalosporins. J Clin Microbiol 1986; 23 (5): 832-9.

46.- Tomasz A, Drugeon H B, de Lencastre H M, Jabes D, McDougall L, Bille J. New mechanism for methicillin resistance in Staphylococcus aureus: clinical isolates that lack the PBP 2a gene and contain normal penicillin-binding proteins with modified penicillin-binding capacity. Antimicrob Agents Chemother 1989; 33 (11): 1869-74. 